Preprint typeset in JHEP style. - PAPER VERSION

\title{
About the $S^{3}$ Group-manifold Reduction of Einstein Gravity
}

\author{
Román Linares-Romero \\ Departamento de Física, Universidad Autónoma Metropolitana Iztapalapa, \\ San Rafael Atlixco 186, c.p. 09340, México D.F., México. \\ E-mail: lirr@xanum.uam.mx
}

\begin{abstract}
We exhibit a new consistent group-manifold reduction of pure Einstein gravity in the vielbein formulation when the compactification group manifold is $S^{3}$. The novel feature in the reduction is to exploit the two 3-dimensional Lie algebras that $S^{3}$ admits. The first algebra is introduced into the group-manifold reduction in the standard way through the Maurer-Cartan 1-forms associated to the symmetry of the general coordinate transformations. The second algebra is associated to the linear adjoint group and it is introduced into the group-manifold reduction through a local transformation in the internal tangent space. We discuss the characteristics of the resulting lower-dimensional theory and we emphasize the novel results generated by the new group-manifold reduction. As an application of the reduction we show that the lower-dimensional theory admits a domain wall solution which upon uplifting to the higher-dimension results to be the self-dual (in the non-vanishing components of both curvature and spin connection) Kaluza-Klein monopole. This discussion may be relevant in the dimensional reductions of $M$-theory, string theory and also in the Bianchi cosmologies in four dimensions.
\end{abstract}

Keywords: Field Theories in Higher Dimensions, Solitons Monopoles and Instantons, Gauge Symmetry. 


\section{Contents}

1. Introduction 1

2. $S^{3}$ group-manifold reduction $\quad 4$

2.1 General coordinate transformations 5

2.2 The adjoint matrix 6

$\begin{array}{lll}2.3 & \text { Parametrization of the vielbein } & 7\end{array}$

2.4 The $D$-dimensional action 9

3. Bianchi type IX domain wall solutions $\quad 10$

3.1 The action and the equations of motion 10

3.2 The self-dual spin connection 12

$\begin{array}{lll}3.3 & \text { First order equations and the superpotential } & 14\end{array}$

4. Conclusions 14

A. Bianchi type IX Lie groups 16

\section{Introduction}

Unification of gravitation and gauge theories in a higher-dimensional theory is one of the most interesting and attractive ideas in physics. If the truly fundamental theory is higherdimensional, then one should be interested in all its predictions and consequences, including solutions. In particular, if one hopes that the 4-dimensional world may be described by the fundamental theory, it is necessary to fully understand the mechanisms (dimensional reductions) to extract lower-dimensional physics from the higher-dimensional theory.

In the dimensional reduction one starts with the curvature scalar (and other possible fields) as the Lagrangian in $D+d$ dimensions. Next one assumes general covariance and supposes that because of some dynamical mechanism, the background manifold $M_{D+d}$ is the direct product of two manifolds: the $d$-dimensional internal compactification manifold $M_{d}$ and the background manifold $M_{D}$ of the resulting $D$-dimensional theory. It turns out that dimensional reductions can be divided into two types $[1,2]$. The first type of reductions consider a quotient space $G / H$ as the internal manifold and are called coset reductions or Pauli reductions [3]. In this paper we shall not discuss this kind of reductions any further.

The second type of reductions are based on the assumption that the parameterizations for the metric and other higher-dimensional fields are invariant under a $d$-dimensional simply transitive acting group of isometries in the internal space. These reductions include both the original Kaluza-Klein reduction on $S^{1}$ in which the group of isometries is 
$U(1)[4,5]$, and the group-manifold reductions where the group of isometries is the left action $\left(G_{d}\right)_{L}$ of the group manifold $G_{d}$. Actually, the metric on the orbit space of the group $G_{d}$ is bi-invariant, i.e. it has $\left(G_{d}\right)_{L} \times\left(G_{d}\right)_{R}$ as its isometry group, but as DeWitt indicated [6], a fully consistent reduction involves a metric which is merely left-invariant. In the literature, the group-manifold reductions are sometimes called DeWitt reductions [6] and sometimes Scherk-Schwarz reductions [7]. All these reductions are consistent because the group invariance of the parametrization ensures that every solution of the lower-dimensional equations of motion corresponds to a solution of the higher-dimensional equations of motion.

Well studied examples of group-manifold reductions are the ones that consider a $(D+$ 3)-dimensional Hilbert-Einstein action as the starting theory and a 3-dimensional internal space invariant under a 3-dimensional group of isometries. In three dimensions the isometry groups are characterized locally by the eleven inequivalent 3 -dimensional Lie algebras $[8$, 9]. In this paper we are interested in dimensional reductions on Bianchi type IX group manifolds, which are defined to be manifolds with an $S O(3)$ or $S U(2)$ isometry group acting transitively on 3-surfaces. Locally both isometry groups are characterized by the same Lie algebra, although topologically they are different, $S U(2)$ is the double covering of $S O(3)$ and they correspond to $S^{3}$ and $\mathbb{R} P^{3}$ respectively $\left(\mathbb{R} P^{3}\right.$ is $S^{3}$ with antipodal points identified).

If one performs the group-manifold reduction of pure Einstein gravity considering to the metric as the basic field, all the geometrical information needed in the reduction is contained in the space-time symmetry. The lower-dimensional theory obtained in this way is an Einstein-Maxwell-scalars gauged theory where the isometry group of the internal space becomes the gauge group of both the Maxwell fields and the scalars of the internal coset space. In the case of Bianchi type IX group manifolds the gauge group of the lower-dimensional theory is either $S O(3)$ or $S U(2)$. For this reason in the literature this dimensional reduction is called $S^{3}=S U(2)$ group-manifold reduction.

Although the metric formulation is appropriate for pure gravity, the presence of spinors requires the introduction of a longer set of variables. These are the vielbein fields which describe local orthonormal Lorentz frames at each space-time point and with respect to which the spinors are defined. In order to treat the group-manifold reduction in the general case is therefore important to perform the reduction of the gravitational sector using the vielbein fields as basic variables. In this formulation gravity has two different local symmetries, the space-time symmetry and the tangent Lorentz symmetry. The standard group-manifold reduction of gravity in the vielbein formulation only exploits the space-time symmetry [7].

The standard Bianchi type IX group-manifold reduction in the vielbein formulation has been applied to many theories, among them, to the 4-dimensional Einstein gravity [10], the $(D+3)$-dimensional Einstein gravity, the bosonic string [1], the 11-dimensional supergravity [11] and to the 10-dimensional supergravity [12,13].

The purpose of this paper is to exhibit a new consistent way to perform the $S^{3}$ groupmanifold reduction of pure Einstein gravity in the vielbein formulation. The novel feature in the reduction is to exploit the two 3-dimensional Lie algebras that the group manifold $S^{3}$ admits. One of the groups is introduced into the group-manifold reduction in the 
standard way through the Maurer-Cartan 1-forms associated to the symmetry of the general coordinate transformations. The another group is dictated by the symmetry of the internal tangent space and it is introduced into the group-manifold reduction through the linear adjoint group $[10,14]$. The role this latter group plays in the spatial topology of the internal manifold has been discussed in [15-18]. We shall show that the introduction of the adjoint matrix $\Lambda$ in the parametrization of the vielbein leads to non trivial differences in the groupmanifold reduction. These differences are: a) A new term in the components of the spin connection with two internal indices and b) an additional term in the covariant derivative of the internal "triad".

We have two main motivations to study this new group-manifold reduction. The first one is related to some results in the context of the 4-dimensional $N=1$ supergravity. Starting with the supersymmetry constraints for full supergravity and then only considering Bianchi type IX homogeneous configurations of the metric an fields, the authors of [17] find: a) There are two distinct definitions of homogeneity and therefore two different Ansätze for the fermion fields and dreibein. One possibility corresponds to the isometry group $S O(3)$ and the another one to $S U(2)$. b) The different Ansätze for the fermion fields depend on whether the spinor components may have the same or opposite sign at antipodal points of the spatial 3-manifold. c) The different Ansätze for the dreibein differ by a orthogonal matrix $\Lambda$ that depends of the internal coordinates. d) The expressions and solutions of the supersymmetry constraints depend on the Ansätze used for the dreibein and the RaritaSchwinger field. For the zero fermion state, the standard definition of homogeneity (without considering $\Lambda$ ) gives rise to a wormhole state [19], whereas the definition of homogeneity involving the matrix $\Lambda$ leads to a Hartle-Hawking state. It is important to stress that although $\Lambda$ is introduced in the Ansätze for the fields in [17], the group-manifold reduction is not performed. In this paper we wish to close this gap.

The second motivation is to get a better understanding of recent results concerning domain wall solutions of 8-dimensional gauged supergravities [20-22] and the relation of these solutions to the classification of 3-dimensional compactification manifolds, both, locally (Bianchi classification [8]) and globally (Thurston classification [23]). The different 8-dimensional gauged supergravities $[11,22]$ arise from group-manifold reductions of the 11-dimensional supergravity [24] over the different 3-dimensional compactification manifolds. In particular the 8-dimensional gauged supergravity of Salam and Sezgin [11] admits $1 / 2$ BPS domain wall solutions which upon uplifting to eleven dimensions become purely gravitational solutions with metrics of the form $\mathbb{R}^{6,1} \times M_{4}$. In this case $M_{4}$ are the selfdual metrics (in both curvature and spin connection) of Belinsky-Gibbons-Page-Pope. It happens that the three equations obtained by require a self-dual spin connection for $M_{4}$ are exactly the same that the ones obtained by require a $1 / 2$ BPS domain wall solution to the 8-dimensional transformation rules for the dilatinos. From the 11-dimensional point of view the uplifted solutions are 1/2 BPS except for an special case which uplift to 11dimensional flat space and hence becomes fully supersymmetric. A disturbing fact is that the Kaluza-Klein monopole [25,26] is also a purely gravitational 1/2 BPS solution of the 11-dimensional supergravity, however by reducing it applying the standard group-manifold reduction, the supersymmetry in eight dimensions becomes fully broken. This happens 
because in the frame of the standard group-manifold reduction the Kaluza-Klein monopole which has the form $\mathbb{R}^{6,1} \times M_{4}$ does not have self-dual spin connection for $M_{4}$.

In this paper we shall exhibit the similarities and differences obtained from the two consistent group-manifold reductions of the $(D+3)$-dimensional Hilbert-Einstein action in the vielbein formulation on Bianchi type IX group manifolds. As an application of the reductions, we study the domain wall solutions of the resulting $D$-dimensional theory and its uplifting to $(D+3)$-dimensions. We shall get the solutions at the level of the first order differential equations that emerge from the self-duality condition of the non-vanishing components of the higher-dimensional spin connection. From the $(D+3)$-dimensional point of view these solutions are of the form $\mathbb{R}^{D-2,1} \times M_{4}$. It is a well known fact that by performing the standard group-manifold reduction, the system of equations obtained by require self-dual spin connection in $M_{4}$ results to be the "Belinsky-Gibbons-Page-Pope" first order system [27]. By performing the new group-manifold reduction we show that the self-duality condition of the spin connection leads to the "Atiyah-Hitchin" first order system [28]. As a consequence the $D$-dimensional theory admits a domain wall solution which upon uplifting to $D+3$ dimensions leads to the self-dual (in both the curvature and the spin connection of $M_{4}$ ) Kaluza-Klein monopole. A preliminary presentation of some of our results can be found in [29]. Here we extend the discussion of the new group-manifold reduction and we show its relation with other well known results in the literature.

The outline of the paper is as follows. In section 2 we perform the $S^{3}$ group-manifold reduction of the $(D+3)$-dimensional Hilbert-Einstein action. We start in 2.1 summarizing the discussion about the group-manifold reduction of the general coordinate transformations given in [7]. In 2.2 we introduce the adjoint matrix $\Lambda$ associated to the group manifold $S^{3}$ whereas in 2.3 we introduce the new parametrization of the vielbein and we compare it with the parametrization of the standard group-manifold reduction. We perform the group-manifold reduction of the spin connection and the action in 2.4. In section 3 we obtain the domain wall solutions of the reduced action. We start analyzing the solutions to the second order differential equations of motion in 3.1 and in 3.2 we discuss the domain wall solutions from the point of view of the self-duality condition of the spin connection. We conclude the section in 3.3 writing down the first-order Bogomol'nyi equations associated to the lower-dimensional action. Our conclusions are given in section 4. In appendix A we explicitly construct the different quantities involved in the reduction.

\section{2. $S^{3}$ group-manifold reduction}

This section will focus on computing the new $S^{3}$ group-manifold reduction of the $(D+3)$ dimensional Hilbert-Einstein action. In the vielbein formulation, Einstein gravity has two local symmetries, the general coordinate symmetry and the tangent Lorentz symmetry. We shall consider the vielbein parametrization in terms of lower-dimensional fields that involves besides the usual 3-dimensional Lie algebra associated to the general coordinate transformations of the internal space another 3-dimensional Lie algebra associated to the local tangent symmetry [17]. 
In the following discussion we assume a $(D+3)$ split of the $(D+3)$ space-time coordinates $x^{\hat{\mu}}=\left(x^{\mu}, z^{\alpha}\right)$ where $\mu=\{0,1, \ldots, D-1\}$ are the indices of the $D$-dimensional space-time and $\alpha=\{1,2,3\}$ are the indices of the internal coordinates. The corresponding flat indices of the tangent space are denoted by $\hat{a}=(a, m)$. The group indices are also denoted with the letters $m, n, \ldots$, . We work in the conventions of [22].

\subsection{General coordinate transformations}

In the vielbein formalism, the $(D+3)$-dimensional Hilbert-Einstein action

$$
S=\int d^{D+3} \hat{x} \hat{e} \hat{\mathcal{R}}(\hat{\omega}),
$$

is invariant under the general coordinate transformations

$$
\delta_{\hat{\mathbf{K}}} \hat{e}_{\hat{\mu}}^{\hat{a}}=\mathcal{L}_{\hat{\mathbf{K}}} \hat{e}_{\hat{\mu}}^{\hat{a}}=\hat{K}^{\hat{\nu}} \partial_{\hat{\nu}} \hat{e}_{\hat{\mu}}^{\hat{a}}+\partial_{\hat{\mu}} \hat{K}^{\hat{\nu}} \hat{e}_{\hat{\nu}}^{\hat{a}} .
$$

As usual, $\hat{e}$ is the determinant of the vielbein, $\hat{\mathcal{R}}$ the Ricci scalar, $\hat{\omega}$ the spin connection and $\mathcal{L}_{\hat{\mathbf{K}}}$ denotes the Lie derivative along the infinitesimal vector field parameters $\hat{\mathbf{K}}$.

As it has been pointed out in [7], the group-manifold reduction is specified by choosing the internal coordinate dependence of the parameters $\hat{K}^{\hat{\mu}}(x, z)$. If they are taken as

$$
\hat{K}^{\mu}(x, z)=K^{\mu}(x), \quad \hat{K}^{\alpha}(x, z)=K^{m}(x)\left(U^{-1}(z)\right)_{m}{ }^{\alpha},
$$

where $U_{\alpha}{ }^{m}(z)$ are $G L(3, \mathbb{R})$ matrices which can be interpreted as the components of the left invariant Maurer-Cartan 1-forms $\sigma^{m} \equiv d z^{\alpha} U_{\alpha}{ }^{m}(z)$, an arbitrary 3-dimensional Lie algebra can be extracted out of the group of general coordinate transformations in $(D+3)$ dimensions. The algebra of general coordinate transformations

$$
\left[\delta_{\hat{K}_{1}}, \delta_{\hat{K}_{2}}\right]=\delta_{\hat{K}_{3}} \quad \text { where } \quad \hat{K}_{3}^{\hat{\mu}}(x, z)=2 \hat{K}_{[2}^{\hat{\nu}}(x, z) \partial_{\hat{\nu}} \hat{K}_{1]}^{\hat{\mu}}(x, z),
$$

gives origin to three different possibilities in $D$-dimensions. First, the algebra of two space-time transformations with parameters $K_{1}^{\mu}(x)$ and $K_{2}^{\mu}(x)$ gives a new space-time transformation with parameter $K_{3}^{\mu}(x)=2 K_{[2}^{\nu}(x) \partial_{\nu} K_{1]}^{\mu}(x)$ indicating that the theory has general coordinate transformations in the $D$-dimensional space-time. Second, the commutator of a space-time transformation with parameter $K_{1}^{\mu}(x)$ and an internal transformation with parameter $K_{2}^{m}(x)$ gives a new internal transformation with parameter $K_{3}^{m}(x)=K_{1}^{\mu}(x) \partial_{\mu} K_{2}^{m}(x)$ which means that the parameters of an internal transformation are space-time scalars. Finally, the commutator of two internal transformations with parameters $K_{1}^{m}(x)$ and $K_{2}^{m}(x)$ produces a new internal transformation with parameter $K_{3}^{p}(x)=f_{m n}^{p} K_{1}^{m}(x) K_{2}^{n}(x)$ where

$$
f_{m n}{ }^{p}=-2\left(U^{-1}(z)\right)_{m}{ }^{\alpha}\left(U^{-1}(z)\right)_{n}{ }^{\beta} \partial_{[\alpha} U_{\beta]}{ }^{p}(z),
$$

are the structure constants of the 3 -dimensional Lie group $G_{3}$, whose Lie algebra $\mathfrak{g}_{3}$ is given by

$$
\left[\mathbf{K}_{m}, \mathbf{K}_{n}\right]=f_{m n}^{p} \mathbf{K}_{p}
$$


and the $f_{m n}{ }^{p}$ 's satisfy the Jacobi identity $f_{[m n}{ }^{q} f_{p] q}{ }^{r}=0$.

After apply the group-manifold reduction $[6,7]$ the simply transitive 3-dimensional Lie algebra (2.6) becomes the algebra of the gauged group in the lower-dimensional theory. It turns out that in three dimensions there exist eleven different ways to choose the structure constants $[8,9]$ and therefore eleven group-manifold reductions [10]. Among them we are interested in the Bianchi type IX group-manifold reductions for which the structure constants are $f_{m n}{ }^{p}=\varepsilon_{m n q} \delta^{p q}$. Bianchi type IX metrics are defined to be manifolds with either an $S O(3)$ or $S U(2)$ isometry group acting on 3-surfaces. Topologically the group $S O(3)$ is the projective space $\mathbb{R} P^{3}$ whilst the group $S U(2)$ is $S^{3}$. The projective space $\mathbb{R} P^{3}$ results from $S^{3}$ by identifying pairs of antipodal points. Explicitly the vectors $\mathbf{K}_{m}$ are given by

$$
\begin{aligned}
& \mathbf{K}_{1}=\frac{\cos z^{3}}{\cos z^{2}} \partial_{1}+\sin z^{3} \partial_{2}-\frac{\cos z^{3} \sin z^{2}}{\cos z^{2}} \partial_{3}, \\
& \mathbf{K}_{2}=-\frac{\sin z^{3}}{\cos z^{2}} \partial_{1}+\cos z^{3} \partial_{2}+\frac{\sin z^{3} \sin z^{2}}{\cos z^{2}} \partial_{3}, \\
& \mathbf{K}_{3}=\partial_{3} .
\end{aligned}
$$

with

$$
0 \leq z^{1} \leq 2 \pi, \quad-\frac{\pi}{2} \leq z^{2} \leq \frac{\pi}{2}
$$

and

$$
\begin{array}{ll}
0 \leq z^{3} \leq 2 \pi, & \text { if } G_{3} \text { is } S O(3), \\
0 \leq z^{3} \leq 4 \pi, & \text { if } G_{3} \text { is } S U(2) .
\end{array}
$$

\subsection{The adjoint matrix}

If we perform the group-manifold reduction of pure gravity using the metric as the basic field, all the geometrical information is codified in the general coordinate transformations. However, if we perform the group-manifold reduction using the vielbein as the basic field, we have two different possibilities for the properties of the fermion components (even if we do not introduce fermions explicitly). They can have either the same or opposite sign at antipodal points of the spatial 3-manifold [17]. These two different possibilities must be codified in the symmetry of the internal tangent space and reflected in geometrical quantities such as the spin connection.

The novel ingredient of the new group-manifold reduction in the vielbein formulation is the introduction of the matrix $\Lambda(z)$, which is taken in the adjoint representation of the 3dimensional Lie algebra (2.6) of the previous section $[10,14]$. The mathematical properties of the linear adjoint group are discussed in detail in [14]. Here, we only point out some properties of the adjoint matrix and in the appendix A we give explicit representations of them.

The adjoint matrix $\Lambda(z)$ is determined by the equation

$$
\Lambda(z)=e^{z^{1} R_{1}} e^{z^{2} R_{2}} e^{z^{3} R_{3}},
$$


where the constant matrices $R_{m}$ are the generators of the Lie algebra $g l(3, \mathbb{R})$ in its natural basis $\left\{\mathbf{e}_{p}{ }^{n}\right\}$, and are given by the adjoint representation of the parameters of the internal general coordinate transformations, $R_{m}=f_{m n}{ }^{p} \mathbf{e}_{p}{ }^{n}=a d_{\mathbf{K}}\left(\mathbf{K}_{m}\right)$. They satisfy the $A D(S U(2))=S O(3)$ Lie algebra

$$
\left[R_{m}, R_{n}\right]=f_{m n}^{p} R_{p}
$$

The relation between the adjoint matrix and the left invariant Maurer-Cartan 1-forms is given by $\boldsymbol{\Lambda}^{-1} d \boldsymbol{\Lambda}=\sigma^{m} R_{m}$. This expression is very important in the group-manifold reduction because it relates the two different matrices that contain the geometrical information of the internal manifold. In components the relation reads

$$
\left(R_{m}\right)_{n}^{p}=\left(U^{-1}(z)\right)_{m}{ }^{\alpha}\left(\Lambda^{-1}(z)\right)_{n}^{q} \partial_{\alpha} \Lambda_{q}^{p}(z) .
$$

It can be shown that the adjoint matrix is orthogonal and also that $\operatorname{det} \Lambda=1$. These properties indicate that $\Lambda(z)$ can be considered as a rotation matrix in the internal tangent space.

\subsection{Parametrization of the vielbein}

The next step in the group-manifold reduction is to make a suitable parametrization of the group-invariant vielbein in terms of lower-dimensional fields. The parametrization includes internal coordinates dependence dictated by the symmetries of the theory. It turns out that for Bianchi type IX group-manifold reductions there are two possibilities to do it. The first possibility has been extensively discussed and only considers internal coordinates dependence through the components $U(z)$ of the Maurer-Cartan 1-forms [7], which are related to the symmetry of the general coordinates transformations. Throughout this paper we refer to this reduction as the standard group-manifold reduction. The second possibility considers besides $U(z)$ a new dependence on the internal coordinates through the adjoint matrix $\Lambda(z)$, which is related to the symmetry of the internal local tangent space. Throughout the paper we refer to this reduction as the "new" group-manifold reduction. The parametrization of the vielbein for the latter possibility is

$$
\hat{e}_{\hat{\mu}} \hat{a}(x, z)=\left(\begin{array}{cc}
e^{c_{1} \varphi(x)} e_{\mu}{ }^{a}(x) & e^{c_{2} \varphi(x)} A_{\mu}{ }^{\alpha}(x, z) L_{\alpha}{ }^{p}(x, z) \\
0 & e^{c_{2} \varphi(x)} L_{\alpha}{ }^{p}(x, z)
\end{array}\right),
$$

where $c_{1}$ and $c_{2}$ are constants whose values are $c_{1}=-\sqrt{3} / \sqrt{2(D+1)(D-2)}$ and $c_{2}=$ $-c_{1}(D-2) / 3^{1}$. The $A_{\mu}$ 's are gauge fields and $L_{\alpha}{ }^{p}(x, z)$ is a $3 \times 3$ matrix whose internal coordinates dependence are given by

$$
\begin{aligned}
A_{\mu}{ }^{\alpha}(x, z) & =A_{\mu}{ }^{m}(x)\left(U^{-1}(z)\right)_{m}{ }^{\alpha}, \\
L_{\alpha}{ }^{p}(x, z) & =U_{\alpha}{ }^{m}(z) L_{m}{ }^{n}(x) \Lambda_{n}{ }^{p}(z) .
\end{aligned}
$$

\footnotetext{
${ }^{1}$ The values of $c_{1}$ and $c_{2}$ ensure that the reduction of the Hilbert-Einstein action yields a pure HilbertEinstein term in $D$-dimensions, with no pre-factor involving the scalar $\varphi$, and that $\varphi$ has a canonically normalized kinetic term in $D$-dimensions.
} 
The vielbein parametrization of the standard group-manifold reduction can be obtained from (2.15) replacing the adjoint matrix $\Lambda$ by the identity matrix. The parametrization of the vielbein can be rewritten in the shorter form

$$
\begin{aligned}
\hat{e}^{a}(x, z) & =e^{c_{1} \varphi(x)} e^{a}(x), \\
\hat{e}^{m}(x, z) & =e^{c_{2} \varphi(x)}\left(A^{n}(x)+\sigma^{n}(z)\right) L_{n}{ }^{p}(x) \Lambda_{p}{ }^{m}(z) \equiv e^{p}(x, z) \Lambda_{p}{ }^{m}(z) .
\end{aligned}
$$

The group-manifold reduction works out because the internal coordinate dependence can be factored out in any geometrical quantity due to the fact that it always appears in one of the two possible combinations (2.5) or (2.12). This means for instance that upon reduction $\delta L_{\alpha}^{p}(x, z)=U_{\alpha}^{m}(z) \delta L_{m}^{n}(x) \Lambda_{n}^{p}(z)$.

From the $D$-dimensional point of view under a space-time transformation $K^{\mu}(x)$, the fields $\varphi(x)$ and $L_{m}{ }^{n}(x)$ transform as scalars whilst $e_{\mu}{ }^{a}(x)$ and $A_{\mu}{ }^{m}(x)$ transform as vectors, and under an internal transformation $K^{m}(x)$, the fields $e_{\mu}{ }^{a}(x)$ and $\varphi(x)$ do not transform whilst the fields $A_{\mu}{ }^{m}(x)$ and $L_{m}{ }^{n}(x)$ transform in the following way

$$
\begin{aligned}
& \delta A_{\mu}{ }^{m}(x)=\left(\partial_{\mu} K^{m}-A_{\mu}{ }^{n} f_{n p}{ }^{m} K^{p}\right), \\
& \delta L_{m}{ }^{n}(x)=\left(f_{m p}{ }^{q} L_{q}{ }^{n}+L_{m}{ }^{q}\left(R_{p}\right)_{q}{ }^{n}\right) K^{p} .
\end{aligned}
$$

The conclusion from the first equation is that the $A_{\mu}{ }^{m}$ 's are gauge potentials for the corresponding gauge group $G_{3}$ whose Lie algebra is (2.6). In (2.19) we have the first consequence due to the introduction of the adjoint matrix $\Lambda$ in the parametrization of the vielbein. Additional to the standard term $f_{m p}{ }^{q} L_{q}{ }^{n}$ originated by the equation (2.5) and related to the gauging of the $S U(2)$ Lie algebra (2.6), we have the new term $L_{m}{ }^{q}\left(R_{p}\right)_{q}{ }^{n}$ originated by the equation (2.12) and related to the $S O(3)$ Lie algebra (2.11). These two terms shall be part of the covariant derivative of the scalar fields $L_{m}{ }^{n}$.

Using the vielbein parametrization (2.13)-(2.15) we can rewrite the 11-dimensional interval in the way

$$
d s^{2}=e^{2 c_{1} \varphi} g_{\mu \nu} d x^{\mu} d x^{\nu}-e^{2 c_{2} \varphi} \mathcal{M}_{m n}\left(d x^{\mu} A_{\mu}{ }^{m}+\sigma^{m}\right)\left(d x^{\nu} A_{\nu}^{n}+\sigma^{n}\right),
$$

where

$$
\mathcal{M}_{m n}(x) \equiv-L_{m}^{p}(x) L_{n}^{q}(x) \eta_{p q} .
$$

In general $L_{m}{ }^{n}(x, z)$ describes the 6 -dimensional $G L(3, \mathbb{R}) / S O(3)$ scalar coset of the internal space and can be interpreted as the internal "triad". It transforms under a global $G L(3, \mathbb{R})$ acting from the left and a local $S O(3)$ symmetry acting from the right. By a gauge fixing of the $\mathrm{SO}(3)$ symmetry, is possible to find an explicit representative of it $[21,22]$. The matrix $\mathcal{M}_{m n}(x)$ is the $S O(3)$ invariant metric of the internal manifold and it is parameterized by the same scalars. In particular, for the case of Bianchi type IX group manifolds the Lie algebra (2.6) corresponds to the algebra of the maximal compact subgroup of $G L(3, \mathbb{R})$ i.e. $S O(3)$ (it is also the Lie algebra of $S U(2)$ ) and the scalar coset $L_{m}{ }^{n}$ is 5-dimensional.

At this point is clear that if we consider quantities that only depend of the internal metric $\mathcal{M}_{m n}$, the local tangent symmetry is irrelevant. This is not the case if we consider geometrical quantities whose definition is given in terms of the triad $L_{m}{ }^{n}$ such as the spin 
connection. The main result of this paper is to realize that is possible to consider the adjoint matrix $\Lambda(z)$ in a consistent group-manifold dimensional reduction scheme.

Upon reduction the independence of the internal coordinates $z^{\alpha}$ is guaranteed because it is factored out in any quantity. Explicitly, if $\hat{T}(x, z)$ is a $(D+3)$-dimensional field, upon reduction for each index $\alpha$ or $m$ that it contains, the internal dependence appears in one of the following ways

$$
\begin{array}{ll}
\hat{T}^{\alpha}(x, z)=t^{m}(x)\left(U^{-1}(z)\right)_{m}{ }^{\alpha}, & \hat{T}_{\alpha}(x, z)=U_{\alpha}{ }^{m}(z) t_{m}(x), \\
\hat{T}^{m}(x, z)=t^{n}(x) \Lambda_{n}{ }^{m}(z), & \hat{T}_{m}(x, z)=\left(\left(\Lambda^{-1}(z)\right)_{m}{ }^{n} t_{n}(x) .\right.
\end{array}
$$

In these expressions $t(x)$ are the corresponding expressions of $\hat{T}$ in the $D$-dimensional space-time. Since in the action all the indices are contracted, the internal dependence vanish.

\subsection{The $D$-dimensional action}

Once discussed the general characteristics of the vielbein parametrization, we apply the $S^{3}$ group-manifold reduction to the $(D+3)$-dimensional Hilbert-Einstein action. The important quantities in the vielbein formalism are the components of the spin connection $\hat{\omega}_{\hat{a} \hat{b}}$. By using the parametrization (2.13)-(2.15), the $(D+3)$-dimensional spin connection reads

$$
\begin{aligned}
& \hat{\omega}_{a b}=\omega_{a b}-2 c_{1} e^{-c_{1} \varphi} \hat{e}_{[a} \partial_{b]} \varphi-\frac{1}{2} e^{\left(c_{2}-2 c_{1}\right) \varphi} F_{a b}{ }^{m} L_{m n} e^{n}, \\
& \hat{\omega}_{a m}=\left(\Lambda^{-1}\right)_{m}{ }^{n}\left[e^{c_{1} \varphi} e^{p}\left(c_{2} \partial_{a} \varphi \eta_{p n}+\left(L^{-1}\right)_{(p}{ }^{q} \mathcal{D}_{a} L_{q \mid n)}\right)+\frac{1}{2} e^{\left(c_{2}-2 c_{1}\right) \varphi} F_{a b}{ }^{p} L_{p n} e^{b}\right], \quad \\
& \hat{\omega}_{m n}=\left(\Lambda^{-1}\right)_{m}{ }^{p}\left(\Lambda^{-1}\right)_{n}{ }^{q}\left[-\hat{e}^{a} e^{-c_{1} \varphi}\left(L^{-1}\right)_{[p}{ }^{r} \mathcal{D}_{a} L_{r \mid q]}+e^{r} e^{-c_{2} \varphi}\left(\mathcal{F}_{r[p q]}-\frac{1}{2} \mathcal{F}_{p q r}+\left(\mathcal{R}_{r}\right)_{p q}\right)\right] .
\end{aligned}
$$

In these expressions $F^{m}=2 \partial A^{m}-f_{n p}{ }^{m} A^{n} A^{p}$ is the gauge vector field strength, the scalar functions $\mathcal{F}$ and $\mathcal{R}$ are defined as

$$
\mathcal{F}_{m n p} \equiv\left(L^{-1}\right)_{m}^{q}\left(L^{-1}\right)_{n}^{r} L_{s p} f_{q r}{ }^{s}, \quad\left(\mathcal{R}_{p}\right)_{m n} \equiv\left(L^{-1}\right)_{p}^{r}\left(R_{r}\right)_{m n}
$$

whereas the covariant derivative of the internal triad is given by

$$
\mathcal{D}_{\mu} L_{m}{ }^{n}=\partial_{\mu} L_{m}{ }^{n}-A_{\mu}^{p} L_{q}^{n} f_{m p}^{q}+A_{\mu}^{p} L_{m}^{q} f_{q p}{ }^{n} .
$$

Notice that as anticipated, the covariant derivative of the internal triad reflects its relation with the two Lie algebras under consideration. The second term corresponds to the standard $S U(2)$ gauging generated by the symmetry of the internal coordinate transformations whereas the third one is related to the symmetry of the internal tangent space.

Using the reduced spin connection it turns out that the reduction of the $(D+3)$ dimensional action is

$$
S=C \int d^{D} x \sqrt{|g|}\left[\mathcal{R}+\frac{1}{4} \operatorname{Tr}\left(\mathcal{D} \mathcal{M} \mathcal{M}^{-1}\right)^{2}+\frac{1}{2}(\partial \varphi)^{2}-\frac{1}{4} e^{-\frac{2 c_{1}}{3}(D+1) \varphi} F^{m} \mathcal{M}_{m n} F^{n}-\mathcal{V}\right]
$$


where $\mathcal{V}$ is the scalar potential

$$
\mathcal{V}=\frac{1}{4} e^{\frac{2 c_{1}}{3}(D+1) \varphi}\left[2 \mathcal{M}^{m n} f_{m p}^{q} f_{n q}^{p}+\mathcal{M}^{m n} \mathcal{M}^{p q} \mathcal{M}_{r s} f_{m p}{ }^{r} f_{n q}{ }^{s}\right],
$$

and $C$ the group volume defined by $C(S U(2))=\int d^{3} z \operatorname{det}\left(U_{\alpha}{ }^{m}\right)=16 \pi^{2}$. In this expression of $C$ we have used the property that $\operatorname{det} \Lambda=1$. From the covariant derivative of the internal triad (2.26), is direct to compute the covariant derivative of the internal metric $\mathcal{M}$

$$
\mathcal{D} \mathcal{M}_{m n}=\partial \mathcal{M}_{m n}+2 f_{q(m}^{p} A^{q} \mathcal{M}_{n) p}
$$

which reflects its invariant character under transformations in the internal tangent space.

In conclusion, the two differences produced by apply the new group-manifold reduction with respect to the standard one are reflected in the extra term $\left(\mathcal{R}_{p}\right)_{m n}$ of the components $\hat{\omega}_{m n}$ of the spin connection (2.24) and in the extra term in the covariant derivative of the internal triad (2.26).

These differences are not manifest in the reduced action and therefore in the equations of motion either because they are enterally written in terms of $\mathcal{M}_{m n}$. The reduced Lagrangian has the same functional form independently of the group-manifold reduction applied (either the standard one or the new one) and therefore the gauge group of the lower-dimensional theory is either $S O(3)$ or $S U(2)$. The result is expected because the difference in the parametrization of the vielbein among both group-manifold reductions is a transformation in the internal tangent space and the internal metric is invariant under such transformation. However the new group-manifold reduction has leaved its imprint in the internal components of the spin connection. In the next section we shall show a consequence of this result at the level of the domain wall solutions of the resulting lower-dimensional theory.

\section{Bianchi type IX domain wall solutions}

In this section we discuss the domain wall solutions to the $D$-dimensional action (2.27). The solutions were originally given for the case $D=1$ and Euclidean signature in [30]. We shall keep in the following discussion the generic dimension $D$.

\subsection{The action and the equations of motion}

After group-manifold reduction the $D$-dimensional field content is $\left\{e_{\mu}{ }^{a}, L_{m}{ }^{n}, \varphi, A^{m}\right\}$. For Bianchi type IX group manifolds the 5-dimensional scalar coset $L_{m}{ }^{n}$ contains two dilatons and three axions. An explicit representation of $L_{m}{ }^{n}$ in terms of the five scalars can be found in $[21,22]$. In order to simplify the discussion is convenient to consider the following consistent truncated parametrization of the scalar coset

$$
L_{m}^{n}(x)=\operatorname{diag}\left(e^{-\frac{\sigma}{\sqrt{3}}}, e^{-\frac{\phi}{2}+\frac{\sigma}{2 \sqrt{3}}}, e^{\frac{\phi}{2}+\frac{\sigma}{2 \sqrt{3}}}\right),
$$

where we have set the axions to zero. In terms of the dilaton fields, the action can be rewritten in the following way

$$
S=C \int d^{D} x \sqrt{|g|}\left[\mathcal{R}+\frac{1}{2}(\partial \phi)^{2}+\frac{1}{2}(\partial \sigma)^{2}+\frac{1}{2}(\partial \varphi)^{2}-\frac{1}{4} e^{-\frac{2 c_{1}}{3}(D+1) \varphi} F^{m} \mathcal{M}_{m n} F^{n}-\mathcal{V}\right],
$$


where

$$
\mathcal{V}=-\frac{1}{4} e^{\frac{2 c_{1}}{3}(D+1) \varphi}\left[e^{\frac{2 \sigma}{\sqrt{3}}}+e^{-\phi-\frac{\sigma}{\sqrt{3}}}+e^{\phi-\frac{\sigma}{\sqrt{3}}}-e^{-\frac{4 \sigma}{\sqrt{3}}}-e^{-2 \phi+\frac{2 \sigma}{\sqrt{3}}}-e^{2 \phi+\frac{2 \sigma}{\sqrt{3}}}\right] .
$$

We are interested in solutions of cohomogeneity one also known as domain wall solutions. These are solutions of the theory in the truncation $A_{\mu}=0$ that only depend on one spatial coordinate orthogonal to the compactification manifold, hence we take the following ansatz

$$
\begin{gathered}
d s_{D}^{2}=f^{2}(y) d x_{(D-1)}^{2}-g^{2}(y) d y^{2}, \\
\varphi=\varphi(y), \quad L_{m}^{n}=L_{m}^{n}(y) .
\end{gathered}
$$

At the beginning due to the ansatz, we have $D+3$ non-trivial second order equations of motion for the fields, $D$ of them corresponding to the diagonal components of the metric tensor $g_{\mu \nu}$ and three corresponding to the scalar fields $\varphi, \phi$ and $\sigma$. However it turns out that only two of the equations of motion for the metric tensor are independent, the ones for $g_{y y}$ and $g_{00}$ (the other $(D-2)$ for $g_{i i}$ are the same as the equation of motion for $\left.g_{00}\right)$. It is direct to show that taking $f(y)=e^{-c_{1} \varphi}$ the equation of motion for $g_{00}$ becomes the same as the equation of motion for the scalar field $\varphi$ reducing the system to four independent equations of motion. By the additional choice $g(y)=e^{\left(3 c_{2}-c_{1}\right) \varphi}$ we can simplify the equations to the form

$$
\begin{gathered}
-e^{2\left(3 c_{2}-c_{1}\right) \varphi} \mathcal{V}=\partial_{y}^{2} \varphi, \quad e^{2\left(3 c_{2}-c_{1}\right) \varphi} \frac{\delta \mathcal{V}}{\delta \phi}=\partial_{y}^{2} \phi, \quad e^{2\left(3 c_{2}-c_{1}\right) \varphi} \frac{\delta \mathcal{V}}{\delta \sigma}=\partial_{y}^{2} \sigma \\
-e^{2\left(3 c_{2}-c_{1}\right) \varphi} \mathcal{V}=\frac{10}{6}\left(\partial_{y} \varphi\right)^{2}+\frac{1}{2}\left(\partial_{y} \sigma\right)^{2}+\frac{1}{2}\left(\partial_{y} \phi\right)^{2}
\end{gathered}
$$

This system of equations was studied long time ago [30] and its solutions are well known. In order to make contact with the original literature we introduce a change of variables in the following way

$$
\varphi=\ln (a b c)^{1 / 3 c_{2}}, \quad \sigma=\ln \left(\frac{b c}{a^{2}}\right)^{1 / \sqrt{3}}, \quad \phi=\ln \left(\frac{c}{b}\right) .
$$

Notice that $a, b$, and $c$ are positive variables. In terms of them the action reads

$$
S \propto \int d y\left[2\left(\frac{\partial_{y} a}{a} \frac{\partial_{y} b}{b}+\frac{\partial_{y} b}{b} \frac{\partial_{y} c}{c}+\frac{\partial_{y} c}{c} \frac{\partial_{y} a}{a}\right)-\frac{1}{2}\left(a^{4}+b^{4}+c^{4}-2 a^{2} b^{2}-2 b^{2} c^{2}-2 c^{2} a^{2}\right)\right] .
$$

The four equations of motion are

$$
2 \partial_{y}^{2}(\ln a)=a^{4}-\left(b^{2}-c^{2}\right)^{2},
$$

plus the two equations obtained by cyclic permutation of $(a, b, c)$ and

$$
4\left(\frac{\partial_{y} a}{a} \frac{\partial_{y} b}{b}+\frac{\partial_{y} b}{b} \frac{\partial_{y} c}{c}+\frac{\partial_{y} c}{c} \frac{\partial_{y} a}{a}\right)=2 a^{2} b^{2}+2 b^{2} c^{2}+2 c^{2} a^{2}-a^{4}-b^{4}-c^{4} .
$$

In these variables the $D$-dimensional interval (3.4) can be rewritten as

$$
d s_{D}^{2}=(a b c)^{-2 c_{1} / 3 c_{2}} d x_{(D-1)}^{2}-(a b c)^{\left(6 c_{2}-2 c_{1}\right) / 3 c_{2}} d y^{2},
$$


and upon uplifting, the $(D+3)$-dimensional interval is of the form $\mathbb{R}^{D-2,1} \times M_{4}$, explicitly

$$
d s_{D+3}^{2}=d x_{D-1}^{2}-\left((a b c)^{2} d y^{2}+a^{2} \sigma_{1}^{2}+b^{2} \sigma_{2}^{2}+c^{2} \sigma_{3}^{2}\right) .
$$

The $D$-dimensional domain wall solutions and the manifolds $M_{4}$ are entirely given by the three positive functions $a(y), b(y)$ and $c(y)$ satisfying the equations (3.8) and (3.9). The solutions describe cohomogeneity one self-dual solutions to the 4-dimensional Euclidean Einstein gravity in empty space.

We are not going to discuss the whole list of manifold solutions $M_{4}$. To our purpose it is enough to mention that some interesting solutions are the BGPP metrics [27], the self-dual Taub-NUT metrics [30,31] and the Eguchi-Hanson metrics [32,33].

\subsection{The self-dual spin connection}

The manifolds $M_{4}$ are self-dual solutions to the 4-dimensional Euclidean Einstein gravity. The self-dual character means that for these manifolds the 4-dimensional curvature is selfdual $\left(\tilde{R}_{I J}=R_{I J}\right)$. It was found that the self-duality condition of the curvature gives origin to second order differential equations of motion that accept two different sets of first integrals [30]. Each set consists of the three equations

$$
2 \frac{\partial_{y} a}{a}=-a^{2}+b^{2}+c^{2}-2 \lambda b c, \quad \text { and cyclic. }
$$

If $\lambda=0$ the set of equations is known as the BGPP system [27], whereas if $\lambda=1$, the set of equations is known as the Atiyah-Hitchin system [28].

As discussed in [33], for the 4-dimensional Euclidean gravity, self-duality in the spin connection is both a sufficient condition for the self-duality of $R_{I J}$ and hence for solving the Einstein equations, and necessary in the sense that if $R_{I J}=\tilde{R}_{I J}$ is satisfied, one can always transform $\omega_{I J}$ by an $O(4)$ gauge transformation into the form $\omega_{I J}=\tilde{\omega}_{I J}$. The advantage to do this is that we deal with first order instead of second order differential equations.

After applying a Bianchi type IX group-manifold reduction to the $(D+3)$-dimensional spin connection and considering the domain wall solution (3.10), we end with six independent non-vanishing components of the spin connection $\left(\hat{\omega}_{\underline{y} m}, \hat{\omega}_{m n}\right)$ which correspond to the components of the spin connection of the manifold $M_{4}$.

We define the dual of the spin connection as

$$
\tilde{\hat{\omega}}_{I J}=\frac{1}{2} \varepsilon_{I J} K L \hat{\omega}_{K L},
$$

where $I, J=\{y, 1,2,3\}$ and $\varepsilon_{y 123}=1$.

By demanding that the spin connection of the metric $M_{4}$ in the basis $\left(a b c d y, a \sigma^{1}, b \sigma^{2}, c \sigma^{3}\right)$ be self-dual we obtain the BGPP system of first order differential equations [27]

$$
2 \frac{\partial_{y} a}{a}=-a^{2}+b^{2}+c^{2}, \quad \text { and cyclic. }
$$

This parametrization of the basis occurs in the standard group-manifold reduction. When the three invariant directions are different, i.e. $a \neq b \neq c$ the equations (3.14) admit the 
BGPP metrics as solutions [27] whilst if two of them are equal i.e. $(a=b \neq c)$ admit the Eguchi-Hanson metrics as solutions [32,33].

If instead we apply the new $S^{3}$ group-manifold reduction, by require a self-dual spin connection we get three independent first order differential equations

$$
\begin{aligned}
c_{2} \partial_{y} \varphi-\left(L^{-1}\right)_{(1}{ }^{p} \partial_{y} L_{p \mid 1)} & =\frac{1}{2} e^{\left(c_{1}-c_{2}\right) \varphi}\left(-\mathcal{F}_{123}+\mathcal{F}_{231}-\mathcal{F}_{312}-2\left(\mathcal{R}_{1}\right)_{23}\right), \\
c_{2} \partial_{y} \varphi-\left(L^{-1}\right)_{(2}{ }^{p} \partial_{y} L_{p \mid 2)} & =\frac{1}{2} e^{\left(c_{1}-c_{2}\right) \varphi}\left(-\mathcal{F}_{123}-\mathcal{F}_{231}+\mathcal{F}_{312}-2\left(\mathcal{R}_{2}\right)_{31}\right), \\
c_{2} \partial_{y} \varphi-\left(L^{-1}\right)_{(3}{ }^{p} \partial_{y} L_{p \mid 3)} & =\frac{1}{2} e^{\left(c_{1}-c_{2}\right) \varphi}\left(+\mathcal{F}_{123}-\mathcal{F}_{231}-\mathcal{F}_{312}-2\left(\mathcal{R}_{3}\right)_{12}\right) .
\end{aligned}
$$

Or in terms of the variables $a, b$ and $c$ we have, the Atiyah-Hitchin first order system

$$
2 \frac{\partial_{y} a}{a}=-a^{2}+b^{2}+c^{2}-2 b c, \quad \text { and cyclic. }
$$

When two of the tree invariant directions are equal i.e. $(a=b \neq c)$ this system admits the Taub-NUT family of metrics as solutions [30]. It is important to stress the origin of the different terms in the r.h.s. of the equation (3.16). The square terms are associated to the $\mathcal{F}$ scalar terms of the internal spin connection, which come from the structure constants of the $S U(2)$ Lie algebra (2.6). The terms like $-2 b c$ are associated to the $\mathcal{R}$ scalar terms and come from the constant matrices $R_{m}$ of the $S O(3)$ Lie algebra (2.11).

This result should not be surprising, as fact in [34] was shown that if the 4-dimensional metric is related to the general class of multi-instantons obtained in [35], the self-duality condition in the spin connection implies that the metric is self-dual Ricci flat. Depending of the election of a constant parameter, the multi-instantons become either the multi TaubNut metrics or the multi Eguchi-Hanson metrics. The same result was obtained in the context of 3-dimensional Toda equations [36].

Now we have a clear picture of the relation between the two different Bianchi type IX group-manifold reductions and the domain wall type solutions of the reduced theory. Because the equations of motion (second order differential equations) are the same in both cases, the domain wall solutions coincide as well. However from the point of view of the first order differential equations, the solutions are divided into two disjoint sets. One set is given by the metrics that solve the BGPP system (3.14) and the another one by the metrics that solve the Atiyah-Hitchin system (3.16). If we reduce pure Einstein gravity applying the standard group-manifold reduction the domain walls that solve the BGPP system are self-dual in both the curvature and the spin connection of $M_{4}$ whereas the metrics in the another set of solutions are self-dual only in the curvature. If instead we reduce applying the "new" group-manifold reduction the conclusion is the opposite. The possibility of relate the different first order systems with the inclusion (or not) of the adjoint matrix $\Lambda$ was already suggested in $[17,37]$.

It is well known that in the case that $a, b$ and $c$ are positive variables, one of the EguchiHanson metrics and one of the Taub-NUT metrics are the only complete non-singular $S O(3)$ hyper-Kähler metrics in four dimensions $[30,38]$, both of them are obtained in the case in which two of the invariant directions are equal. From the $(D+3)$-dimensional point of 
view these two solutions correspond to $\mathbb{R}^{D-2,1} \times M_{4}$ with either $M_{4}$ the Eguchi-Hanson metric [32] whose generic orbits are $\mathbb{R} P^{3}$ [27] or the self-dual Taub-NUT solution whose generic orbits are $S^{3}$ [31]. In the latter case, the complete $(D+3)$-metric is known as the Kaluza-Klein monopole $[25,26]$.

\subsection{First order equations and the superpotential}

As established in [34], the Lagrangian of the action (3.7) can be written as

$$
L=T-V=\frac{1}{2} g_{m n}\left(\frac{\partial \alpha_{m}}{\partial y}\right)\left(\frac{\partial \alpha_{n}}{\partial y}\right)+\frac{1}{2} g^{m n}\left(\frac{\partial W}{\partial \alpha_{m}}\right)\left(\frac{\partial W}{\partial \alpha_{n}}\right)
$$

where $\alpha_{m} \equiv(\ln a, \ln b, \ln c)$ and $W$ is a superpotential given by

$$
W=a^{2}+b^{2}+c^{2}-2 \lambda b c-2 \lambda c a-2 \lambda a b .
$$

We conclude that the case $\lambda=0$ is related to the standard group-manifold reduction whereas the case $\lambda=1$ is related to the new group-manifold reduction. In the literature concerning domain wall solutions is usual to write down the superpotential in terms of the original variables, i.e. in terms of the dilatons. The inverse variable transformations of (3.6) are

$$
a(y) \equiv e^{c_{2} \varphi-\frac{\sigma}{\sqrt{3}}}, \quad b(y) \equiv e^{c_{2} \varphi+\frac{\sigma}{2 \sqrt{3}}-\frac{\phi}{2}}, \quad c(y) \equiv e^{c_{2} \varphi+\frac{\sigma}{2 \sqrt{3}}+\frac{\phi}{2}} .
$$

It is straightforward to show that in terms of the superpotential and the dilatons, the potential satisfies the property

$$
V=\frac{1}{2}\left(\left(\frac{\partial W}{\partial \varphi}\right)^{2}+\left(\frac{\partial W}{\partial \phi}\right)^{2}+\left(\frac{\partial W}{\partial \sigma}\right)^{2}-\left(\frac{D-1}{D+1}\right) W^{2}\right) .
$$

It is also possible to write down the BGPP first order system (3.14) and the Atiyah-Hitchin first order system (3.16) in terms of the dilatons and the superpotential. The equations in this case become

$$
\frac{\partial \varphi}{\partial y}=\frac{1}{6 c_{2}} W, \quad \frac{\partial \phi}{\partial y}=-\frac{\partial W}{\partial \phi}, \quad \frac{\partial \sigma}{\partial y}=-\frac{\partial W}{\partial \sigma},
$$

which are related with first-order Bogomol'nyi equations (see for example [39-42] and references therein).

\section{Conclusions}

In this paper we have introduced a new consistent $S^{3}$ group-manifold reduction of Einstein pure gravity in the vielbein formulation by considering the two 3-dimensional Lie algebras that $S^{3}$ admits. We have showed that although the lower-dimensional theory has the same Lagrangian independently of the Bianchi type IX group-manifold reduction used (either the standard one or the new one), there exist two differences produced by apply the new group-manifold reduction with respect to the standard one. These differences are a) A 
new term in the components of the spin connection with two internal indices and b) an additional term in the covariant derivative of the internal triad.

As an application of these results we studied the domain wall solutions of the lowerdimensional theory and we concluded that because the equations of motion coincide for both Bianchi type IX group-manifold reductions, the domain wall solutions coincide as well. Upon uplifting to the higher-dimension these are purely gravitational solutions of the form $\mathbb{R}^{D-2,1} \times M_{4}$. However from the point of view of first order differential equations, the solutions are divided into two disjoints sets, one set is given by the metrics that solve the BGPP system and the another set by the metrics that solve the Atiyah-Hitchin system. If we reduce pure Einstein gravity applying the standard group-manifold reduction the domain walls that solve the BGPP system are self-dual in both the curvature and the spin connection of $M_{4}$ whereas the metrics in the another set of solutions are self-dual only in the curvature. If instead we reduce applying the new group-manifold reduction the conclusion is the opposite, i.e. now the domain walls that solve the Atiyah-Hitchin system are self-dual in both the curvature and the spin connection of $M_{4}$ whilst metrics in the another set of solutions are self-dual only in the curvature. This result can be relevant if we are working with a formulation of gravity in which the fundamental field is the vielbein instead of the metric, for instance, in supergravity.

We believe the results of this paper open the possibility to construct an 8-dimensional gauged supergravity by apply the new $S^{3}$ group-manifold reduction to the 11-dimensional supergravity. The hope is that this supergravity could admit a $1 / 2$ BPS domain wall solution which upon uplifting to eleven dimensions led to the Kaluza-Klein monopole. This problem should be analogous to the results obtained in the context of the 4-dimensional $N=1$ supergravity $[17,19]$. In this case the non-singular solution $\mathbb{R}^{6,1} \times M_{4}$ of the 11-dimensional supergravity should be the analogous of the wormhole state if $M_{4}$ is the Eguchi-Hanson metric [32] and of the Hartle-Hawking state if $M_{4}$ is the self-dual Taub-NUT solution [31]. It would be interesting to establish this analogy explicitly.

Usually when the internal orthonormal frame specified by $e^{m}$ is rotated by a $z$ dependent orthogonal transformation $e^{m} \rightarrow e^{n} \lambda_{n}{ }^{m}(z)$, the change in the corresponding spin connection is interpreted as a gauge transformation because the spin connection transforms exactly like a Yang-Mills potential [33]. It would be interesting to see whether the local adjoint matrix $\Lambda(z)$ can be interpreted as a kind of large gauge transformation, at least for some special type of solutions. This interpretation is suggested because the adjoint matrix changes the expression of the spin connection in such a way that when we impose the condition of self-duality, we obtain two different systems of first order differential equations that accept solutions with isometry groups of different topology.

The tools used in this paper are not exclusive to 3-dimensional group-manifolds and it would be interesting to see whether the generalization to other dimensions is possible [14].

\section{Acknowledgments}

We are grateful to Eric Bergshoeff, Ulf Gran, Hugo Morales, Mikkel Nielsen, Tomás Ortín 
and Diederik Roest for interesting and useful discussions at different stages of this work. In particular we should like to thank Mikkel Nielsen, Tomás Ortín and Diederik Roest for a critic reading to an early version of the draft. Special thanks to Emily and Pepe for encourage me to work in this project each time it had gone. We also would like to thank the Centre for Theoretical Physics at University of Groningen and the Instituto de Ciencias Nucleares at Universidad Nacional Autónoma de México for hospitality and financial support at different stages of this work. This research was supported in part by the project CONACyT-40745-F.

\section{A. Bianchi type IX Lie groups}

In the next discussion we give explicit expressions for the relevant quantities used in the $S^{3}$ group-manifold reduction, we follow the conventions of [14]. The starting point is to assume a 3 -dimensional vector fields basis $\mathbf{K}_{m}$ that satisfies the Lie algebra $\mathfrak{g}_{3}$

$$
\left[\mathbf{K}_{m}, \mathbf{K}_{n}\right]=f_{m n}{ }^{p} \mathbf{K}_{p} .
$$

In the Bianchi type IX case the expression for the structure constants can be diagonalized and taken as

$$
f_{m n}^{p}=\epsilon_{m n q} \delta^{q p}, \quad \delta^{m n}=\operatorname{diag}(1,1,1) .
$$

Choosing the matrices $\left\{\mathbf{e}_{m}{ }^{n}\right\}$ as the basis of $\mathfrak{s l}(3, \mathbb{R})$ where $\mathbf{e}_{m}{ }^{n}$ is the matrix whose only non-vanishing component is a one in the $m^{\text {th }}$ row and $n^{\text {th }}$ column, the canonical basis $\left\{R_{m}\right\}$ of the canonical adjoint group $A d_{\mathbf{K}}(G)$ is defined as the adjoint representation of the generators $\mathbf{K}$ in this basis, $\left(R_{m}\right)=f_{m n}{ }^{p} \mathbf{e}_{p}{ }^{n}$, explicitly

$$
\left(R_{1}\right)_{m}{ }^{n}=\left(\begin{array}{ccc}
0 & 0 & 0 \\
0 & 0 & -1 \\
0 & 1 & 0
\end{array}\right), \quad\left(R_{2}\right)_{m}{ }^{n}=\left(\begin{array}{rrr}
0 & 0 & 1 \\
0 & 0 & 0 \\
-1 & 0 & 0
\end{array}\right), \quad\left(R_{3}\right)_{m}{ }^{n}=\left(\begin{array}{ccc}
0 & -1 & 0 \\
1 & 0 & 0 \\
0 & 0 & 0
\end{array}\right),
$$

and satisfy the algebra

$$
\left[R_{m}, R_{n}\right]=f_{m n}^{p} R_{p}
$$

Exponentiating the generators of the Lie algebra $\mathfrak{g}_{3}$ in the adjoint representation, we get the adjoint representation $\Lambda(z)$ of the group $G_{3}$

$$
\Lambda_{m}^{n}(z)=e^{z^{1} R_{1}} e^{z^{2} R_{2}} e^{z^{3} R_{3}}=\left(\begin{array}{ccc}
c_{2} c_{3} & -c_{2} s_{3} & s_{2} \\
c_{1} s_{3}+c_{3} s_{1} s_{2} & c_{3} c_{1}-s_{1} s_{2} s_{3} & -s_{1} c_{2} \\
s_{1} s_{3}-c_{3} c_{1} s_{2} & c_{3} s_{1}+c_{1} s_{2} s_{3} & c_{1} c_{2}
\end{array}\right)
$$

where we have used the following abbreviations $(a=1,2,3)$

$$
c_{a} \equiv \cos z^{a}, \quad s_{a} \equiv \sin z^{a} .
$$


It can be directly checked that $\operatorname{det} \Lambda=1$ and also that the matrix is orthogonal $\Lambda_{m}{ }^{p}(z) \Lambda_{n}{ }^{q}(z) \eta_{p q}=\eta_{m n}$. The next step is to compute the left invariant 1-forms using the equation $\boldsymbol{\Lambda}^{-1} d \boldsymbol{\Lambda}=\sigma^{m} R_{m}$. Its dual base $\left\{\mathbf{K}_{m}\right\}$ can also be obtained by require $\sigma^{m} \mathbf{K}_{n}=\delta_{m}^{n}$.

$$
\begin{aligned}
\sigma^{1} & =\cos z^{2} \cos z^{3} d z^{1}+\sin z^{3} d z^{2}, & \mathbf{K}_{1} & =\frac{\cos z^{3}}{\cos z^{2}} \partial_{1}+\sin z^{3} \partial_{2}-\frac{\cos z^{3} \sin z^{2}}{\cos z^{2}} \partial_{3}, \\
\sigma^{2} & =-\cos z^{2} \sin z^{3} d z^{1}+\cos z^{3} d z^{2}, & \mathbf{K}_{2} & =-\frac{\sin z^{3}}{\cos z^{2}} \partial_{1}+\cos z^{3} \partial_{2}+\frac{\sin z^{3} \sin z^{2}}{\cos z^{2}} \partial_{3}, \\
\sigma^{3} & =\sin z^{2} d z^{1}+d z^{3}, & \mathbf{K}_{3} & =\partial_{3} .
\end{aligned}
$$

From these expressions we have that the matrix $U_{\alpha}{ }^{m}(z)$ is given by

$$
U_{\alpha}{ }^{m}(z)=\left(\begin{array}{ccc}
\cos z^{2} \cos z^{3} & -\cos z^{2} \sin z^{3} & \sin z^{2} \\
\sin z^{3} & \cos z^{3} & 0 \\
0 & 0 & 1
\end{array}\right) .
$$

The relation between the left and right invariant Lie algebras, and the relation between the left and right invariant 1 -forms is

$$
\tilde{\mathbf{K}}_{m}=\Lambda_{m}{ }^{n} \mathbf{K}_{n}, \quad \quad \tilde{\sigma}^{m}=\sigma^{n}\left(\Lambda^{-1}\right)_{n}{ }^{m} .
$$

Using them we get

$$
\begin{array}{ll}
\tilde{\sigma}^{1}=\sin z^{2} d z^{3}+d z^{1}, & \tilde{\mathbf{K}}_{1}=\partial_{1}, \\
\tilde{\sigma}^{2}=-\cos z^{2} \sin z^{1} d z^{3}+\cos z^{1} d z^{2}, & \tilde{\mathbf{K}}_{2}=-\frac{\sin z^{1}}{\cos z^{2}} \partial_{3}+\cos z^{1} \partial_{2}+\frac{\sin z^{1} \sin z^{2}}{\cos z^{2}} \partial_{1}, \\
\tilde{\sigma}^{3}=\cos z^{2} \cos z^{1} d z^{3}+\sin z^{1} d z^{2}, & \tilde{\mathbf{K}}_{3}=\frac{\cos z^{1}}{\cos z^{2}} \partial_{3}+\sin z^{1} \partial_{2}-\frac{\cos z^{1} \sin z^{2}}{\cos z^{2}} \partial_{1} .
\end{array}
$$

All these quantities satisfy the Lie algebra $\mathfrak{g}(\tilde{\mathfrak{g}})$

$$
\left[\mathbf{K}_{m}, \mathbf{K}_{n}\right]=f_{m n}{ }^{p} \mathbf{K}_{p}, \quad\left[\tilde{\mathbf{K}}_{m}, \tilde{\mathbf{K}}_{n}\right]=-f_{m n}{ }^{p} \tilde{\mathbf{K}}_{p}, \quad\left[\mathbf{K}_{m}, \tilde{\mathbf{K}}_{n}\right]=0 .
$$

and the Maurer-Cartan equations

$$
d \sigma^{m}=-\frac{1}{2} f_{n p}^{m} \sigma^{n} \wedge \sigma^{p}, \quad d \tilde{\sigma}^{m}=\frac{1}{2} f_{n p}{ }^{m} \tilde{\sigma}^{n} \wedge \tilde{\sigma}^{p} .
$$

\section{References}

[1] M. Cvetic, G. W. Gibbons, H. Lu and C. N. Pope, Consistent group and coset reductions of the bosonic string, Class. Quant. Grav. 20 (2003) 5161, hep-th/0306043.

[2] G. W. Gibbons and C. N. Pope, Consistent $S^{2}$ Pauli reduction of six-dimensional chiral gauged Einstein-Maxwell supergravity, Nucl. Phys. B697 (2004) 225, hep-th/0307052.

[3] N. Straumann, On Pauli's invention of non-Abelian Kaluza-Klein theory in 1953, gr-qc/0012054. 
[4] T. Kaluza, On the problem of unity in physics, Sitzungsber. Preuss. Akad. Wiss. Berlin (Math. Phys.) K1 (1921) 966.

[5] O. Klein, Quantum theory and five-dimensional theory of relativity, Z. Phys. 37 (1926) 895.

[6] B. S. DeWitt, Dynamical theory of groups and fields, in Relativity, groups and topology, Les Houches 1963 (Gordon and Breach, 1964) 585.

[7] J. Scherk and J. H. Schwarz, How to get masses from extra dimensions, Nucl. Phys. B153 (1979) 61.

[8] L. Bianchi, Sugli spazi a tre dimensioni che ammettono un gruppo continuo di movimenti, Tomo XI (1898) 267, For an English translation see: On the three-dimensional spaces which admit a continuous group of motions, Gen. Rel. and Grav. 33 (2001), 2171.

[9] F. B. Estabrook, H. D. Wahlquist and C. G. Behr, Dyadic analysis of spatial homogeneous world models, J. Math. Phys. 9 (1968) 497.

[10] G. F. R. Ellis and M. A. H. MacCallum, A class of homogeneous cosmological models, Commun. Math. Phys. 12 (1969) 108.

[11] A. Salam and E. Sezgin, D = 8 supergravity, Nucl. Phys. B258 (1985) 284.

[12] A. H. Chamseddine and M. S. Volkov, Non-Abelian BPS monopoles in $N=4$ gauged supergravity, Phys. Rev. Lett. 79 (1997) 3343, hep-th/9707176.

[13] A. H. Chamseddine and W. A. Sabra, $D=7$ SU(2) gauged supergravity from $D=10$ supergravity, Phys. Lett. B476 (2000) 415, hep-th/9911180.

[14] R. T. Jantzen, The dynamical degrees of freedom in spatially homogeneous cosmology, Commun. Math. Phys. 64 (1979) 211.

[15] J. F. Luciani, Space-time geometry and symmetry breaking, Nucl. Phys. B135 (1978) 111.

[16] A. Asthekar and J. Samuel, Bianchi Cosmologies: The role of spatial topology, Class. Quant. Grav. 8 (1991) 2191.

[17] R. Graham and H. Luckock, The Hartle-Hawking state for the Bianchi IX model in supergravity, Phys. Rev. D49 (1994) 4981, gr-qc/9311004.

[18] T. Koike, M. Tanimoto and A. Hosoya, Compact homogeneous universes, J. Math. Phys. 35 (1994) 4855, gr-qc/9405052.

[19] P. D. D'Eath, Quantization of the Bianchi IX model in supergravity, Phys. Rev. D48 (1993) 713.

[20] R. Hernandez and K. Sfetsos, An eight-dimensional approach to G(2) manifolds, Phys. Lett. B536 (2002) 294-304, hep-th/0202135.

[21] N. Alonso-Alberca, E. Bergshoeff, U. Gran, R. Linares, T. Ortín and D. Roest, Domain walls of $D=8$ gauged supergravities and their $D=11$ origin, JHEP 06 (2003) 038, hep-th/0303113.

[22] E. Bergshoeff, U. Gran, R. Linares, M. Nielsen, T. Ortín and D. Roest, The Bianchi classification of maximal $D=8$ gauged supergravities, Class. Quant. Grav. 20 (2003) 3997, hep-th/0306179. Gauged supergravities from Bianchi's group manifolds, Class. Quant. Grav. 21 (2004) S1501. The Bianchi classification of maximal D=8 gauged supergravities, Fortsch. Phys. 52 (2004) 472.

[23] W. P. Thurston, Three-dimensional geometry and topology, Princeton University Press (1997). 
[24] E. Cremmer, B. Julia and J. Scherk, Supergravity theory in 11 dimensions, Phys. Lett. B76 (1978) 409.

[25] R. Sorkin, Kaluza-Klein monopole, Phys. Rev. Lett. 51 (1983) 87.

[26] D. J. Gross and M. J. Perry, Magnetic monopoles in Kaluza-Klein theories, Nucl. Phys. B226 (1983) 29.

[27] V. Belinskii, G. Gibbons, D. Page and C. Pope, Asymptotically euclidean Bianchi IX metrics in quantum gravity, Phys. Lett. B76 (1978) 433.

[28] M. F. Atiyah and N. J. Hitchin, Low-energy scattering of nonabelian monopoles, Phys. Lett. A107 (1985) 21.

[29] R. Linares, Three-dimensional group manifold reductions of gravity, AIP Conference Proceedings 758 (2005) 208; $S^{3}$ group-manifold reduction of gravity, To appear in proceedings of the VI Mexican School (DGFM), Approaches to Quantum Gravity.

[30] G. Gibbons and C. Pope, The positive action conjecture and asymptotically Euclidean metrics in quantum gravity, Commun. Math. Phys. 66 (1979) 267.

[31] S. W. Hawking, Gravitational instantons, Phys. Lett. A60 (1977) 81.

[32] T. Eguchi and A. Hansson, Asymptotically flat self-dual solutions to euclidean gravity, Phys. Lett. B74 (1978) 249.

[33] T. Eguchi and A. Hansson, Selfdual solutions to Euclidean gravity, Annals Phys. 120 (1979) 249.

[34] G. W. Gibbons, H. Lu, C. N. Pope and K. S. Stelle, Supersymmetric domain walls from metrics of special holonomy, Nucl. Phys. B623 (2002) 3, hep-th/0108191.

[35] G. W. Gibbons and S. W. Hawking, Gravitational multi-instantons, Phys. Lett. B78 (1978) 430.

[36] R. Hernández and K. Sfetsos, Unpublished work.

[37] K. Sfetsos, Non-Abelian duality, parafermions and supersymmetry, Phys. Rev. D 54 (1996) 1682, hep-th/9602179.

[38] I. Bakas and K. Sfetsos, Toda fields of SO(3) hyper-Kahler metrics and free field realization, Int. J. Mod. Phys. A 12 (1997) 2585, hep-th/9604003.

[39] M. Cvetic, F. Quevedo and S.-J. Rey, Stringy domain walls and target space modular invariance, Phys. Rev. Lett. 67 (1991) 1836.

[40] K. Skenderis and P. K. Townsend, Gravitational stability and renormalization-group flow, Phys. Lett. B468 (1999) 46, hep-th/9909070.

[41] I. Bakas, A. Brandhuber and K. Sfetsos, Domain walls of gauged supergravity, M-branes, and algebraic curves, Adv. Theor. Math. Phys. 3 (1999) 1657, hep-th/9912132.

[42] E. Bergshoeff, M. Nielsen and D. Roest, The domain walls of gauged maximal supergravities and their M-theory origin, JHEP 07 (2004) 006, hep-th/0404100. 